EPiC Series in Engineering
Volume 3, 2018, Pages 1859-1867
HIC 2018. 13th International
Conference on Hydroinformatics

\title{
The Water Tariff in a WDS Rehabilitation
}

\author{
Santopietro S. ${ }^{1}$, Tricarico C. ${ }^{1}$, Morley M. S. ${ }^{2}$, Savic D. A. ${ }^{3}$, \\ Kapelan Z. ${ }^{3}$, Gargano R. ${ }^{1}$ \\ ${ }^{1}$ University of Cassino and Southern Lazio Via G. di Biasio 43, Cassino 03043, Italy \\ ${ }^{2}$ KWR Water Groningenhaven, 73433 PE Nieuwegein, Netherlands \\ ${ }^{3}$ University of Exeter, Exeter, UK \\ s.santopietro@unicas.it, c.tricarico@unicas.it, \\ Mark.Morley@kwrwater.nl, d.savic@exeter.ac.uk, \\ Z.Kapelan@exeter.ac.uk, gargano@unicas.it
}

\begin{abstract}
The aging process in water distribution system is one of the main drivers for the reduction of performances in water distribution systems. Furthermore, this conditions usually imply an increase in the maintenance costs that the water utilities have to sustain. In this situation it is required to undertake programmatic strategies in order to guarantee the highest benefit for both the final users and the water companies. An optimal rehabilitation strategy has been proposed in order to take into account the regulatory schemes that have to be abided at the national level, in the water industry.
\end{abstract}

\section{Introduction}

In the last decades, there has been a change in the structure of the whole water industry, mainly shifting the focus from the only satisfaction of the needs of end users, according to the increasingly higher standards that modern society was dictating, to the ability of the whole industry of relying on itself. In the early ' 90 s there had been increasing awareness on the decay of Water Distribution Systems (WDS) and the financial unsustainability in particular in the International Conference on Water and Sustainable Development (ICWE) held in Dublin was officially recognized that water has an economic value. Such acknowledgement had a deep impact on the regulatory policies of the water industry. A set of reforms were introduced in the legal framework connected to the water sector, directed to actualize a transition from the old public systems to private ones specially regulated schemes.

The principles of tariff design that arose in that period are now well established in many modern countries and regulate in detail the financial aspects of the water utilities. There are several constraints that the tariff structure has to abide and usually there are institutions in charge of the supervision. This regulatory transition not always has been managed in the best possible way, sometimes imposing regulations that were difficult to sustain, in order to protect the final users. 
These scenarios often caused imbalances in the management of water utilities making difficult to find adequate funding to maintain and improve the level of service of WDS.

In scientific literature it is possible to find many works on the optimization and rehabilitation of WDSs (e.g. [1]-[3]) but not many consider an in-depth characterization of the economic framework in which water utilities operate(e.g. [4]). The aim of this study has been to develop a methodology to implement optimal rehabilitation strategies in a staged fashion. Considering a predefined time horizon for the realization of the rehabilitation process, the goal is to find the optimal replacement schedule in order to improve the level of service of the WDS, compatibly with the limits imposed by tariff regulatory frameworks.

To perform this an extensive, yet simplified, financial model has been developed in order to take into consideration how the engineering choices connected to the rehabilitation process, reflected on the water companies' financial layout. Indeed, precisely on the latter the yearly tariff articulation has to be obtained. The optimization has been performed with the Non Sorting Genetic Algorithm II (NSGA-II, [5]) which is a notorious and robust algorithm widely employed in the optimization of WDSs.

\section{Methodology}

\subsection{The Italian water tariff system}

The tariff system in force until 2019 in Italy is the MTI-2, it contemplates a price cap mechanism based on financial information of the water utilities, that they are required to send to the regulator (AEEGSI). The method is based on the average tariff that the water company can charge to the users in the year $a$ (from 2016 to 2019). For each year the considered average tariff is obtained multiplying the tariff of previous years (for MTI-2 is the one from 2015) by a multiplier $\vartheta_{a}$.

$\vartheta_{a}=\frac{V R G_{a}}{T_{2015}}$

$V R G_{a}=$ Capex $_{a}+$ Opex $_{a}+$ FoNI $_{a}+E R C_{a}$

The multiplier $\vartheta_{a}$ as shown in Eq.(1) is calculated as the ratio between the $V R G_{a}$ (Eq.(2))which is the acknowledged revenue of the water company considering the standard costs according to the tariff system and the full-cost recovery principle. The main costs categories for the $a^{\text {th }}$ year, are the following: capital costs $\left(\right.$ Capex $\left._{a}\right)$, operational expenditures $\left(\right.$ Opex $\left._{a}\right)$, a fund for future investments $\left(F o N I_{a}\right)$ and the environmental costs $\left(E R C_{a}\right)$.

The tariff Method is characterized by a dynamic structure through a price limit that depends on intrinsic parameters that describe the economic situation that each water company is facing. There are three criteria to classify a water company: (1) current infrastructures available to the water company, (2) if the water company is in a transition phase (e.g. merging, acquisitions), (3) the per capita operational expenditures compared to the mean values for the national industry.

The multiplier $\vartheta_{a}$ is subject to a variation constraint from year to year expressed by Eq.(3).

$\frac{\vartheta_{a}}{\vartheta_{a-1}} \leq\left[1+r p i+\left(1+\gamma_{K}\right) K-\left(1+\gamma_{X}\right) X\right]$

Where $r p i$ is the inflation rate, $K$ is the price limit assumed to be $5 \%$ and $X$ is the sharing factor which is equal to $0.5 \%$, and $\gamma_{K}$ and $\gamma_{X}$ are multipliers depending on the water company situation according to the three criteria explained above. 


\subsection{Financial model}

When considering the pricing models in regulated markets like in natural monopolies, probably the most established is the Full Cost Recovery. In the European Union it has been introduced in the WFD, in particular in article 9 where it is stated: Member States shall take account of the principle of recovery of the costs of water services, including environmental and resource costs. With this approach all the expenses that have to be sustained, in order to produce the required volume of the resource, have to be recovered through tariff and resource pricing.

In the business jargon, the total expenditures met by a company are usually divided in two categories: Capital expenditure (Capex) and Operational Expenditures (Opex). The first one, Capex, represents the funds used by a company to acquire or upgrade physical assets such as property, industrial buildings or equipment. This type of outlay is also made by companies to maintain or increase the scope of their operations. An operating expense is an expense that a business incurs through its normal business operations. Often abbreviated as OPEX, operating expenses include rent, equipment, inventory costs, marketing, payroll, insurance and funds allocated toward research and development.

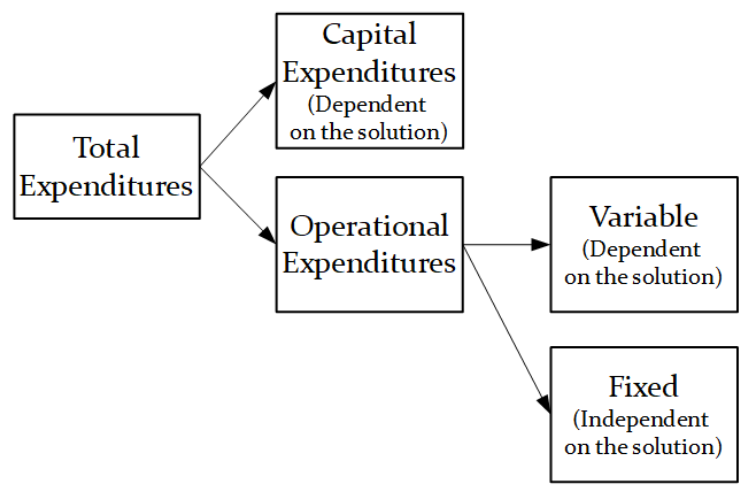

Figure 1: Block diagram for the cost model.

\section{Capex}

Capital expenditures are related to the costs connected to rehabilitation interventions, in other words to pipes' replacement. For each intervention, the cost is calculated multiplying the unit cost, which depends on the diameter and on the material, by the pipe length.

$C_{j}^{s}=C_{u}(D) L_{j}$

To compute the annual capex value, for each intervention has to be considered the annual depreciation expense which have to be summed up.

Capex $_{i}=\sum_{j=1}^{p} C_{j}^{s} q_{a j}$

Where $C_{j}^{S}$ is the replacement cost of pipe $j$ and $q_{i j}$ is the depreciation expense of year $a$ for the replacement of pipe $j$. 


\section{Opex}

The operational expenditures are considered to be compound by two portions. The first one, opex ${ }_{i}^{f i x}$, is independent on the rehabilitation process. This quota is considered to be constant for the entire time horizon and is calculated as a function of the total served users $N_{u s}$.

In order to estimate this relation, a sample of 33 Italian water companies has been analysed (data source: Utilitatis). The total number of supplied users ranges from 70000 to 4 million. This dataset was deduced from a form that water utility companies have to fill according with their accounting and economic information, from their balance sheet, and send it to the local and then national regulation authority.

The document is structured according to the water utilities balance sheet entries and it is divided in two sections, the first one is about production units and revenues and the second one is about expenses. In this elaboration the second part has been considered and in particular all the expenses that are not directly connected to the production itself, but those categories of costs that usually are classified as overhead.

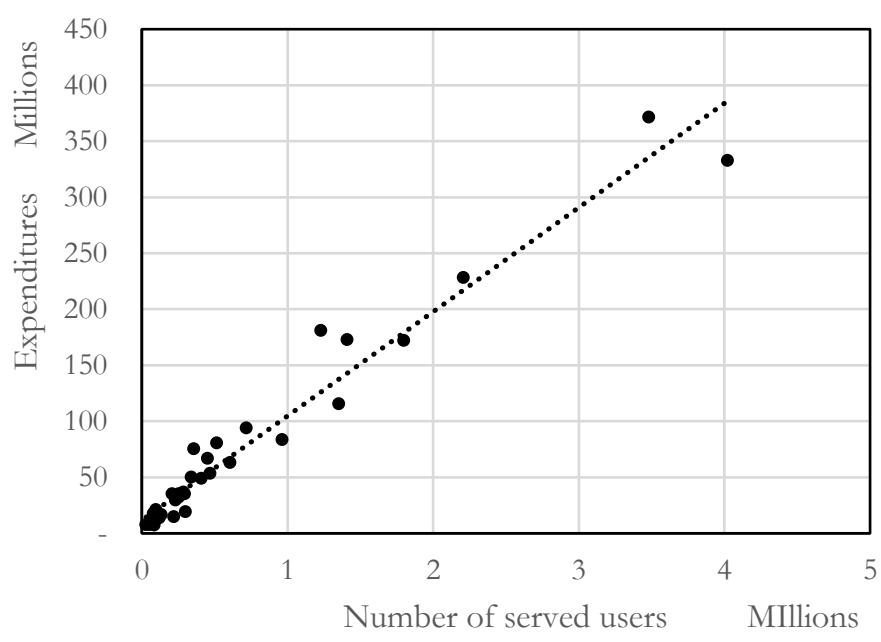

Figure 2: Linear regression of the Italian water utilities data

The regression line of Figure 2, described by Eq.(6), presents a more than acceptable $R^{2}$ score of 0.954 and has an intercept value of roughly $10 \mathrm{M} €$.

Opex ${ }^{\text {fix }}=93 \mathrm{~N}_{U s}+10^{7}$

The variable component of the operational expenditures $O p e x_{i}^{v a r}$ is considered dependent on the expected number of failures of the network pipes. The failure rate of a pipe, considering a deterministic approach, is driven mainly by its age. Many works (eg.,[6] [7]) considered an exponential growth of pipes failure with time, expressed in the following Eq. (7).

$N^{f}(t)=N^{f}\left(t_{0}\right) e^{A\left(t-t_{0}\right)}$

Where $N^{f}(t)$ is the expected number of failures for a pipe at time $t, N^{f}\left(t_{0}\right)$ is the expected number of failures for a pipe at time $t_{0}$ and $\mathrm{A}$ is the growth rate.

The total endogenous component of the operational expenditure for each year is calculated as follows 
Opex $_{a}^{\text {var }}=\sum_{a=1}^{n} \sum_{j=1}^{p} N_{a j}^{f} C_{j}^{r}$

Where $a=$ considered year, $\mathrm{j}=$ considered pipe, $n=$ number of years in the time horizon, $p=$ total number of pipes subject to rehabilitation, $N_{i j}^{f}=$ number of expected failures for pipe $\mathrm{j}$ in year i and $C_{j}^{r}=$ cost of repair for pipe $\mathrm{j}$.

So, the total expenditure due to maintenance and running costs is given by the following

Opex $_{a}=$ opex $_{a}^{\text {var }}+$ opex $_{a}^{\text {fix }}$

\section{Tariff calculation}

According to the total cost recovery framework, the tariff has to be calculated yearly, based on the total expenses incurred in each particular year.

$T_{a}=\frac{\text { Capex }_{a}+\text { Opex }_{a}}{W_{a}}$

Where $W_{i}$ represents the total volume of revenue water for year $i$.

Within almost every national regulatory scheme, to preserve customers from uncontrolled rise of prices, a price cap is considered. This implies that water prices from one year to the next, cannot be increased more than a fixed percentage $(K)$.

$\frac{T_{a+1}}{T_{a}}<K$

\subsection{Optimization framework}

The main goal of this work is to find the optimum pipe replacement schedule for a WDS respecting regulatory schemes that water utilities have to abide. For this purpose, an approach has been developed based on an optimization process followed by a stochastic postprocessing, considering demand variability.

Two objective functions have been considered to take into account the performances of the solutions inside the genetic algorithm. The two main aspects considered to be optimized are: the economy and hydraulic performances.

\section{Net present value}

To evaluate the cost of the overall project in terms of expenses and savings from the reduction of the expected number of breakings, the net present value (NPV) has been considered

$N P V=\sum_{a=0}^{n} \frac{C F_{a}}{(1+r)^{a}}$

Where $r$ is the interest rate, at the considered year, $n=$ number of years of the considered time span and $C F$ is given by the following.

$C F=I_{a}+O p e x_{a}^{v a r}$

Where I is the investment of year a and Opex end is given by Eq.(8).

\section{Hydraulic reliability}

The main goal of a rehabilitation process is to increase the WDS performances. Above all, the objective is to guarantee to all the supplied users an adequate level of service, in line with modern 
society standards. This translates in the system ability of meeting the users' demand. Several authors introduced

Several authors (e.g. [8], [9]) define the local Hydraulic Performance Index (HPI) for day $d$ and in node $j$ (HPI $d$ ) is defined by

$H P I_{i}=\frac{\sum_{k=1}^{n} \alpha_{k, j}^{d} Q_{k, j}^{d} \Delta t}{\sum_{k=1}^{N} Q_{k, j}^{d} \Delta t}$

where $n=$ number of intervals into which the day is divided; $k=$ generic interval of the day $(k=1$, $2, \ldots, n) ; \Delta t=$ time step for which a flow value is considered to be constant; $Q_{k, j}^{d}=$ flow rate demand in node $j$ during the $k$ th $\Delta t$ on day $d ; \alpha_{k, j}^{d}=$ piezometric head availability coefficient for node $j$ in the $k$ th time interval on day $d$.

$\alpha_{k, j}^{d}=1 \quad$ if $\quad H_{k, j}^{d}>\overline{H_{J}}$

$\alpha_{k, j}^{d}=\frac{H_{k, j}^{d}-\widetilde{H_{J}}}{\overline{H_{J}}-\widetilde{H_{J}}} \quad$ if $\quad \overline{H_{J}} \geq H_{k, j}^{d} \geq \widetilde{H_{J}}$

$\alpha_{k, j}^{d}=0 \quad$ if $\quad \widetilde{H_{J}}>H_{k, j}^{d}$

where $H_{k, j}^{d}=$ head in node $j$ during the $k$ th time interval of day $d ; H j=$ minimum head needed to fully satisfy demand at the node $j ; H j=$ elevation of the user in the lowest site out of all those served by node $j$.

The global performance of the entire network is given as weighted average of the nodal HPIs, using as weights the ratio between the daily mean flow required by the users at node $j \hat{Q}_{j}$ and the daily mean of the whole flow required by all the users served by the WDS, $\sum_{j=1}^{N} \hat{Q}_{j}$.

$H P I_{n e t}=\sum_{j=1}^{N} H P I_{j} \frac{\hat{Q}_{j}}{\sum_{j=1}^{N} \hat{Q}_{j}}$

Since the time horizon is compound by more than one year here we consider the average HPI among the considered years.

$\overline{H P I}_{\text {net }}=\sum_{a=1}^{N} H P I_{\text {net }}^{a}$

Finally the considered objective functions are expressed in Eq.(18).

$\{\max \overline{H P I}$

$\{\min N P V$

Subject to the constraints

$\frac{T_{a+1}}{T_{a}}<1.05 \quad \forall i \in[1, n]$

\section{The case study}

The proposed methodology was applied to a case study of a real water distribution network. The case study has been fist presented by [10] and then utilized also by [11] as a benchmark problem for the test of various multi-objective optimization approaches. This case study is particularly interesting for this application, due to its topological dimensions and the overall served demand, resulting in an intermediate problem. As it is understandable, considering the aim of the proposed methodology, the 
considered system has to be well sized in order to represent the system size that a water utility usually have to manage.

The network topology is shown in Figure 3. The network presents three reservoirs, 68 nodes and 99 pipes.

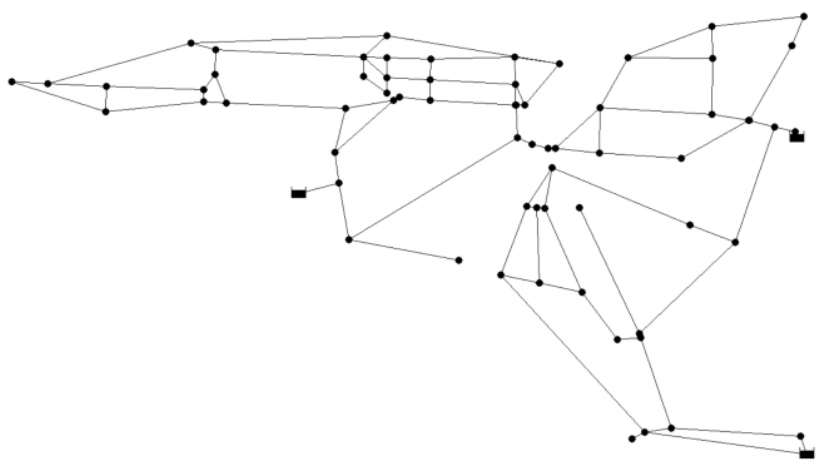

Figure 3: Pescara WDN topology

As starting point an initial tariff $T_{0}=1.6 € / \mathrm{m}^{3}$ has been considered, being it a good estimate for the average national prices.

In Figure 4 is shown the non-dominated pareto front. As we can observe the two objectives present a distinguishable trade-off so to solutions with higher mean hydraulic reliability (HPI) correspond higher costs. The solutions start with an HPI index of roughly 0.88 and as expected never reach the unity. It is worth noting that this doesn't mean that no solution contemplates a HPI index equal to 1, in fact considering as objective function the mean over the time horizon, it is possible that the solution obtains the maximum reliability in the last year.

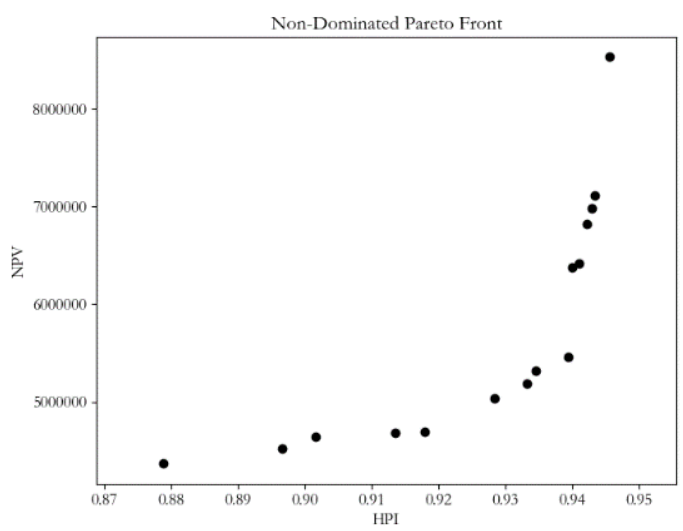

Figure 4: Final pareto front

For each point (solution) of the pareto front is it possible to calculate the financial indicators previously introduced and that concur year by year to the calculation of the water tariff.

As it is sown in Figure 5-a we can see that the considered pipes replacement causes an increase of the Capex, namely the depreciated replacement cost and a reduction of the annual expected repair costs. It is worth noting that the Capex diagram, as it is expected, it is monotonically increasing. This is deeply connected with the total annual expense presented in figure Figure 5-b, in fact the first represent the 
summation of the amortized quotas of the second. The depreciation process smoothens the real expenses from an accounting point of view.
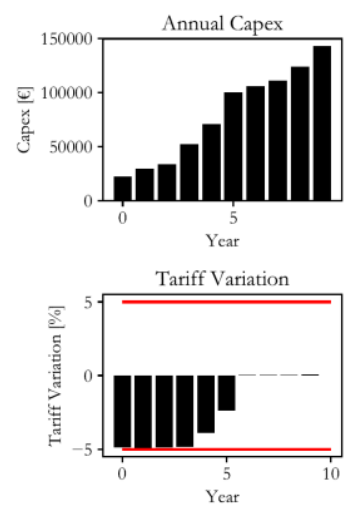

a.

Figure 5: Financial indicators for a sample solution

b.
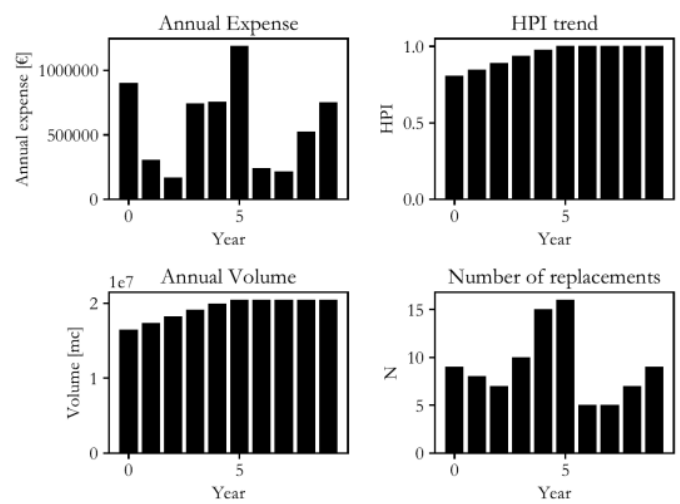

We can observe that the tariff variation for each year is contained within the feasibility bounds, represented by the horizontal red lines. It is interesting to note that for the considered conditions, the tariff is monotonically decreasing throughout the planning horizon. This might be strange to conceive, but it is explained considering the annual revenue volume of Figure 5-b. In fact, with increasing hydraulic reliability the users' water request is more likely to be fulfilled and thus the total water volume increases. Evidently in this particular analyzed problem the rate at which the volume increases, is higher than the rate at which the expenses increase, and thus the tariff reduces in the considered time horizon.

\section{Conclusions}

From the early 2000s, Full Cost Recovery has become the main regulatory standard in Europe. According to this framework the financial performances of the water companies are taken into account to define the water tariff, which is subject to stringent variation constraints.

The presented approach aims to perform a staged rehabilitation process, that each year considers the effects that the incurred costs have on the financial situation of the water company and specifically on the tariff, which is subject to variation constraints. Considering an unconstrained problem, it would lead us to find solutions that are very performing, but practically unfeasible.

In order to consider the economic aspects connected with the tariff in WDSs rehabilitation processes, it has been developed a simplified cost model. Given the direct correlation that incurs between the yearly total expenditures and the tariff, it is necessary to understand how the interventions, driven by hydraulic considerations, affect the economic and financial indicators of the water company. The tariff has to be calculated year by year, with the purpose of covering the total expenses and the latter thus, cannot exceed values that would cause variations in the tariff incompatible whit the price limits.

The application of the proposed approach to a literature case study has shown that it is possible to evaluate the economic indicators of the managing water utility year by year in the analyzed time horizon. This allows to better understand how a particular solution affects the overall economic layout and thus leading to more aware business decisions. 


\section{Acknowledgements}

The Authors would like to acknowledge the Utilitatis foundation for kindly providing the dataset used in this study.

\section{References}

[1] D. A. Savic and G. A. Walters, "Genetic Algorithms for Least-Cost Design of Water Distribution Networks," Journal of Water Resources Planning and Management, vol. 123, no. 2, pp. 67-77, Mar. 1997.

[2] D. Halhal, G. A. Walters, D. A. Savic, and D. Ouazar, "Scheduling of water distribution system rehabilitation using structured messy genetic algorithms," Evolutionary computation, vol. 7, no. 3, pp. 311-329, 1999.

[3] M. Engelhardt, P. Skipworth, D. A. Savic, A. Cashman, G. A. Walters, and A. J. Saul, "Determining maintenance requirements of a water distribution network using whole life costing," Journal of Quality in Maintenance Engineering, vol. 8, no. 2, pp. 152-164, Jun. 2002.

[4] G. C. Dandy, E. A. McBean, and B. G. Hutchinson, "A model for constrained optimum water pricing and capacity expansion,” Water Resources Research, vol. 20, no. 5, pp. 511-520, 1984.

[5] K. Deb, A. Pratap, S. Agarwal, and T. Meyarivan, "A fast and elitist multiobjective genetic algorithm: NSGA-II," Evolutionary Computation, IEEE Transactions on, vol. 6, no. 2, pp. 182-197, 2002.

[6] U. Shamir and C. Howard, "An analytical approach to scheduling pipe replacement," JournalAmerican Water Works Association, vol. 71, no. 5, pp. 248-258, 1979.

[7] T. M. Walski and A. Pelliccia, "Economic analysis of water main breaks," Journal (American Water Works Association), pp. 140-147, 1982.

[8] R. Gargano and D. Pianese, "Reliability as Tool for Hydraulic Network Planning," Journal of Hydraulic Engineering, vol. 126, no. 5, pp. 354-364, 2000.

[9] R. Gupta and P. R. Bhave, "Reliability-Based Design of Water-Distribution Systems," Journal of Environmental Engineering, vol. 122, no. 1, pp. 51-54, Jan. 1996.

[10] C. Bragalli, C. D'Ambrosio, J. Lee, A. Lodi, and P. Toth, “On the optimal design of water distribution networks: a practical MINLP approach,” Optimization and Engineering, vol. 13, no. 2, pp. 219-246, Jun. 2012.

[11] Q. Wang, M. Guidolin, D. Savic, and Z. Kapelan, "Two-objective design of benchmark problems of a water distribution system via MOEAs: Towards the best-known approximation of the true Pareto front," Journal of Water Resources Planning and Management, vol. 141, no. 3, p. 04014060, 2014. 